\title{
Re: Autophagy in Cancer Stem Cells: A Potential Link Between Chemoresistance, Recurrence and Metastasis
}

\section{Ojha R1, Bhattacharyya S2, Singh SK1}

1 Post Graduate Institute of Medical Education and Research, Clinic of Urology, Chandigarh, India

2Post Graduate Institute of Medical Education and Research, Clinic of Biophysics, Chandigarh, India

Biores Open Access 2015;4:97-108. doi: 10.1089/biores.2014.0035.

\section{EDITORIAL COMMENT}

Autophagy is a homeostatic, catabolic degradation process whereby cellular proteins and organelles are engulfed by autophagasomes, digested in lysosomes and recycled to sustain cellular metabolism. Autophagy has dual roles in cancer development. Generally, cancer cells activate autophagy for energy production and can enable cell survival. As well known, a small number of cancer stem cells (CSCs) able to self-renew, repopulate a tumor after treatment, and initiate metastatic growth. Inhibition of autophagy (i.e. 3-methyladenine, wortmannin, antimalarial drugs chloroquine and hydroxychloroquine, LY294002, bafilomycin, monensin) restored chemosensitivity and enhanced tumor cell death. A major regulator of autophagy is the mammalian target of rapamycin (mTOR) pathway which consists of two distinct signaling complexes known as mTORC1 and mTORC2. mTOR is activated downstream of PI3K-AKT, a pathway that is commonly dysregulated in human cancer. It can be inhibited by many therapeutics via this pathway. Autophagy is a predominant factor that helps in the acquisition of resistance to chemotherapy. Some authors reported that stress-induced autophagy in CSCs help their survival and, inhibition of autophagy can overcome CSC resistance. In the context of this knowledge, autophagy can be a novel therapeutic target. Targeting autophagy in cancer will provide new opportunities for drug development in the near future.

Fehmi Narter, MD 